\title{
Les trypanocides et leur utilisation en médecine vétérinaire
}

\author{
par S. M. TOURE (*)
}

\begin{abstract}
RESUME
De nombreuses espèces de trypanosomes peuvent parasiter les animaux domestiques et le traitement ou la protection de ces animaux fait appel à des médicaments divers dont les indications respectives sont mentionnées pour les courants. L'utilisation de ces médicaments peut entraîner à la longue la chimiorésistance de souches de trypanosomes et cette possibilité doit particulièrement retenir l'attention du thérapeute. Enfin, pour tout traitement d'animaux, il conviendra de tenir compte des espèces de trypanosomes à combattre, des drogues à préférer compte tenu de l'état physiologique des animaux. Le présent rapport ne renferme à dessein, qu'un nombre très limité de références bibliographiques traitant de généralités sur la question.
\end{abstract}

\section{INTRODUCTION}

Les trypanosomoses des animaux domestiques sont causées par des espèces de trypanosomes assez nombreuses qui appartiennent, du point de vue systématique, à plusieurs groupes biologiques correspondant à des sous-genres différents. Les espèces pathogènes le plus fréquemment rencontrées chez les animaux sont les suivantes :

1. dans le groupe de Trypanosoma vivax ou sous-genre Duttonella:

a) T. vivax ZIEMANN, 1905 ; parasite les Ruminants et les Equidés.

b) T. uniforme BRUCE et al., 1911 ; signalée chez les Ruminants.

2. dans le groupe de Trypanosoma congolense ou sous-genre Nannomonas:

a) $T$. congolense BRODEN, 1904 ; parasite des Ruminants, des Carnivores et des Equidés.

b) T. simiae BRUCE et al., 1912; le Porc est le plus sensible à cette espèce.

3. dans le groupe de Trypanosoma brucei ou sous-genre Trypanozoon:

a) $T$. brucei PLIMMER et BRADFORD, 1899; très pathogène pour les Equidés et les Carnivores, moins pour les Ruminants.

b) T. evansi STEEL, 1885; pathogène pour le Chameau, mais les Bovins, le Cheval et le Chien sont aussi sensibles à l'espèce.

c) T. equiperdum DOFLEIN, 1901; transmise par contagion, seulement chez le Cheval.

4. une espèce monotypique du sous-genre Pycnomonas : $T$. suis OCHMANN, 1905 qui parasite exclusivement le Porc et les Suidés sauvages.

${ }^{(*)}$ Institut d'Elevage et de Médecine vétérinaire des Pays tropicaux, Laboratoire national de l'Elevage et de Recherches vétérinaires, B.P. 2057, Dakar, Sénégal. 
La diversité des espèces pathogènes de trypanosomes et les différences constatées dans leur virulence suivant les espèces animales parasitées se traduisent, en matière de traitement, par des différences de sensibilité à l'égard des trypanocides. En outre, les animaux ont une susceptibilité variable selon leur espèce et selon les trypanocides qu'on leur administre. Et enfin suivant le médicament employé, son mode d'administration et la fréquence de son utilisation sur le terrain, il y a assez souvent création de souches de trypanosomes qui résistent à un ou plusieurs trypanocides. Tout cela est pour rendre assez complexes les problèmes de thérapeutique dans les trypanosomoses animales. Toutefois, la médecine vétérinaire dispose d'un arsenal thérapeutique sensiblement plus varié qu'en médecine humaine, ce qui permet le plus souvent de trouver une médication appropriée dans les différents cas que le vétérinaire est amené à rencontrer.

Les trypanocides utilisés en médecine vétérinaire peuvent être classés suivant des groupes chimiques divers:

- dérivés de l'urée;

- dérivés de la quinoléine;

- dérivés diamidines;

- dérivés phénanthridinium.

Depuis les travaux du chimiste allemand Paul EHRLICH (1854-1915) (in MULLIGAN, 1970) à qui l'on doit la synthèse des premiers médicaments trypanocides, obtenus à partir de colorants synthétiques, dont la Suramine (1916-1920) et les expériences pratiquées à l'Institut Pasteur de Paris par l'équipe de LAVERAN et MESNIL, à qui l'on doit l'utilisation de la Tryparsamide (1919-1925), jusqu'à nos jours, plusieurs dizaines de composés chimiques ont été employés pour lutter contre les trypanosomoses animales, mais seuls quelques-uns ont survécu et sont utilisables.

\section{LES TRYPANOCIDES D'INTERET VETERINAIRE ET LEURS INDICATONS}

\section{Dérivés de l'urée : la Suramine (synonymes: Moranyl, Naganol, Antrypol)}

La Suramine est utilisée chez les animaux domestiques avec des résultats variables selon l'espèce animale traitée et les parasites en cause. Son efficacité est faible chez les Bovins souffrant de trypanosomose à $T$. vivax ou à $T$. congolense. Par contre, on obtient souvent de bons résultats chez le Dromadaire, le Cheval et le Chien lorsqu'ils sont parasités par une des espèces du groupe de $T$. brucei.

La Suramine est administrée par voie intraveineuse à raison de 7 à $10 \mathrm{mg}$ par $\mathrm{kg}$ de la solution à 10 p. 100 . Deux à trois traitements à intervalle d'une semaine permettent souvent de guérir les animaux dans les cas de trypanosomose à $T$. evansi ou $T$. brucei, chez le Chameau ou le Cheval. Le médicament, administré une seule fois à la dose indiquée, peut aussi protéger ces animaux contre ces infections pendant un à trois mois. La chimio-résistance à la Suramine peut se manifester après administration répétée de suramine à faible dose. Les souches résistantes de $T$. evansi sont sensibles sinon à l'Antrycide du moins au Bérénil.

La tolérance du médicament est assez bonne. Cependant on peut quelquefois noter des réactions générales après son administration intraveineuse. Par voie sous-cutanée ou intramusculaire, on observe inflammation et nécrose locales. L'utilisation prolongée de la Suramine provoque souvent une néphrite chronique avec albuminurie. Chez le Cheval, il y a quelquefois apparition d'œdème, d'urticaire et de fourbure, consécutive à l'emploi du produit.

La Suramine peut être associée à l'Anthiomaline ou au Novarsénobenzol et l'on réalise ainsi une synergie médicamenteuse permettant de guérir plus rapidement et plus sûrement les animaux; chez l'Homme, on associe volontiers Suramine et Tryparsamide.

La Suramine donne naissance, par combinaison avec d'autres trypanocides, à de nombreux complexes, actifs contre les trypanosomes, de faible toxicité générale et ayant un pouvoir pro- 
phylactique très élevé mais presque tous sont interdits à l'usage du fait des réactions sévères provoquées au lieu d'injection (S.C. ou I.M.). On peut citer les complexes Suramine-Pentamidine, Suramine-Quinapyramine, Suramine-Métamidium, Suramine-Ethidium, Suramine-Prothidium. Seul est utilisé, mais assez exceptionnellement, le complexe Suramine-Quinapyramine qui est un des rares trypanocides actifs dans les infections du Porc à $T$. simiae.

\section{Dérivés de la Quinoléine}

\section{a) La Quinapyramine ou Antrycide}

Deux sels de Quinapyramine sont utilisés: le méthylsulfate et le chlorure dont quelques unes des propriétés sont assez dissemblables.

Le méthylsulfate de Quinapyramine est assez soluble dans l'eau (dans la proportion de 33 p. 100) et forme une solution laiteuse. Injectée par voie sous-cutanée, la solution est rapidement absorbée et une forte concentration du médicament dans l'organisme est atteinte en peu de temps; des manifestations de toxicité peuvent apparaître très vite chez l'animal traité.

Le chlorure de Quinapyramine est très peu soluble dans l'eau $(0,12$ p. 100) et forme une suspension dense. Après injection sous-cutanée de la suspension, il se forme localement un dépôt qui n'est que très lentement résorbé. On n'obtient que de faibles concentrations dans le sang et la toxicité du produit est assez faible.

Les deux sels semblent avoir une égale activité trypanocide une fois qu'ils arrivent en contact avec les trypanosomes. Les différences dans leurs propriétés pharmacologiques permettent cependant de les utiliser dans des buts différents : le méthylsulfate, parce qu'il se concentre rapidement dans le sang, est un bon médicament curatif; le chlorure, parce qu'il forme un dépôt sous la peau, est un bon agent préventif.

On appelle Antrycide-prosalt un mélange des deux sels de Quinapyramine, à raison de $3 \mathrm{~g}$ de méthylsulfate pour $4 \mathrm{~g}$ de chlorure, utilisé seulement pour la chimioprophylaxie; l'un des deux sels établit rapidement dans le sang une concentration optimale dont l'autre assure la persistance.

Sur le plan toxicologique, des accidents peuvent se produire avec le méthylsulfate de Quinapyramine à $7 \mathrm{mg} / \mathrm{kg}$ chez les Bovins. La quantité maximale de méthylsulfate seul ou de méthylsulfate contenu dans le prosalt qui pourra être administrée par voie sous-cutanée ou intramusculaire est de $5 \mathrm{mg} / \mathrm{kg}$ chez toutes les espèces animales. Même à cette dose, les signes de souffrance ne sont pas rares: salivation intense, trémor, sudation, inappétence, quelquefois collapsus transitoire, dus aux propriétés curarisantes de l'Antrycide. Chez les Bovins, ces symptômes rétrocèdent rapidement en quelques heures à 24 heures. Par contre, le Cheval et le Chien sont sensibles à la Quinapyramine et il faut prendre soin de fragmenter la dose thérapeutique et l'administrer en deux ou trois fois à 6 heures d'intervalle.

Chez tous les animaux, il y a possibilité d'inflammation adémateuse au lieu d'injection qui se résorbe rapidement pour le méthylsulfate mais se transforme en nodule réactionnel renfermant le dépôt médicamenteux quand il s'agit du chlorure. Ce nodule peut subir une involution scléreuse et demeurer permanent, sans rapport aucun avec la persistance de l'activité trypanocide.

Les sels de Quinapyramine ont une action marquée sur la plupart des trypanosomes: $T$. vivax, $T$. congolense, $T$. brucei et $T$. evansi, et les font disparaître du sang en 24 heures environ. La dose d'utilisation est de 3 à $5 \mathrm{mg} / \mathrm{kg}$ (suivant l'espèce animale) de méthylsulfate en solution à 10 p. 100 , par voie sous-cutanée, à titre curatif et $12 \mathrm{mg} / \mathrm{kg}$ de suspension de prosalt à $24 \mathrm{p}$. 100 par voie sous-cutanée à titre prophylactique. Les infections à $T$. simiae chez le Porc sont passibles d'un traitement par le chlorure de Quinapyramine à $50 \mathrm{mg} / \mathrm{kg}$ mais souvent la maladie est très aiguë et foudroie l'animal avant l'action du médicament.

Depuis leur vulgarisation dans les premières années 1950, les sels d'Antrycide ont été très largement utilisés un peu partout en Afrique et il en est résulté cette conséquence fâcheuse que dans un grand nombre de pays ils sont devenus inopérants sur les trypanosomes du fait d'une chimio-résistance tenace. 
Les trypanosomes résistants à la Quinapyramine sont sensibles à l'Isométamidium sinon au Bérénil.

b) Le Tozocide

Décrit par AUSTIN et al., 1957, le Tozocide est voisin de l'Antrycide. Considéré plus actif que celui-ci expérimentalement, sa toxicité nettement plus grande n'autorise pas cependant son utilisation.

\section{Diamidines trypanocides}

\section{a) La Lomidine}

Elle est utilisée en médecine vétérinaire uniquement pour soigner les piroplasmoses. Alors que ce médicament est l'un des plus importants dans le traitement et la prophylaxie des trypanosomoses humaines, par contre son efficacité dans les trypanosomoses animales est très faible.

\section{b) L'Acéturate de Dinamizène ou Bérénil}

Ce médicament a des propriétés curatives remarquables. Il est très actif contre les infections à $T$. vivax ou à $T$. congolense. Une seule administration à la dose de $3,5 \mathrm{mg} / \mathrm{kg}$ entraîne généralement la guérison des animaux infectés par ces espèces. Les infections à $T$. brucei peuvent être vaincues chez le Cheval et chez les Bovins par une dose de $7 \mathrm{mg} / \mathrm{kg}$ et celles à $T$. evansi chez le Chameau sont passibles d'un traitement à $3,5 \mathrm{mg} / \mathrm{kg}$, sans dépasser cette dose car le Chameau semble sensible à des doses plus élevées. Les trypanosomes disparaissent totalement de la circulation au bout de 12 à 36 heures après son administration. Il est à noter également que le Bérénil est actif dans les cas de piroplasmoses et sur certains germes microbiens tels que les Brucella et les streptocoques. A l'actif du produit, d'autres faits intéressants sont à citer : la rareté d'apparition de chimio-résistance des trypanosomes à son égard et la sensibilité des souches qui sont devenues résistantes à d'autres trypanocides. C'est en maintes occasions le médicament de choix pour combattre la chimio-résistance à l'égard de la Quinapyramine et surtout des dérivés Phénanthridinium.

La toxicité du Bérénil est relativement faible par rapport aux autres trypanocides modernes. A la dose habituelle de $3,5 \mathrm{mg}$ par $\mathrm{kg}$ de la solution à $7 \mathrm{p} .100$ et par voie intramusculaire, le produit est toléré par la plupart des animaux. Des cas d'encéphalopathie œéémateuse ou hémorragique ont quelquefois été signalés à l'autopsie de chiens ayant reçu le Bérénil à raison de $3,5 \mathrm{mg} / \mathrm{kg}$ (14), cependant la plupart des chiens supportent un traitement à $7 \mathrm{mg} / \mathrm{kg}$ et même au-delà.

Chez les vaches laitières, la médication par le Bérénil n'a pas d'influence sur la production; le médicament ne passe pas dans le lait, il est éliminé dans les urines. On peut traiter les femelles gestantes sans crainte de suites fâcheuses.

La chimio-résistance au Bérénil n'est pas courante. Quelques cas de résistance ont été rapportés de Nigéria $(9,13)$ et d'Uganda $(12)$. La résistance au Bérénil peut être vaincue par les dérivés Phénanthridinium.

\section{Trypanocides dérivés de la Phénanthridine}

\section{a) Le Dimidium}

Il existe deux sels de Dimidium, le bromure et le chlorure. Dans la pratique, seul le chlorure est utilisable.

Le Bromure de Dimidium, quoique très actif sur les trypanosomes des animaux et jadis utilisé, provoque des réactions sévères au point d'injection et présente pour les Herbivores des propriétés photosensibilisantes inacceptables dans la pratique. De plus, sa toxicité générale est assez élevée. 
Le Chlorure de Dimidium est, par contre, moins toxique, encore que l'on observe quelquetois des manifestations tardives de photosensibilisation avec nécrose hépatique périportale plusieurs semaines après son administration à des animaux de robe claire qui transhument sous un soleil ardent. Son activité trypanocide et ses propriétés toniques et eutrophiques l'ont cependant accrédité pendant longtemps auprès de nombreux agents vétérinaires.

Le Chlorure de Dimidium est administré à raison de 0,8 à $1 \mathrm{mg} / \mathrm{kg}$ de la solution à 1 ou 2 p. 100, par voie intraveineuse lente. A cette dose, T. vivax et $T$. congolense sont généralement sensibles. Dans les infections chroniques à $T$. vivax, il y a cependant assez souvent rechute à $1 \mathrm{mg} / \mathrm{kg}$ et les doses plus élevées sont dangereuses, surtout chez le Cheval. Le pouvoir protecteur du médicament est faible : 20 à 30 jours avec une dose de 0,8 à $1 \mathrm{mg} / \mathrm{kg}$.

Des souches résistantes de trypanosomes peuvent être créées à l'usage du Dimidium et il y a possibilité de résistance croisée avec les autres dérivés Phénanthridinium. Cette résistance peut être vaincue par le Bérénil.

\section{b) L'Ethidium ou Homidium}

L'Ethidium a été réalisé en 1952 par WATKINS et WOOLFE par substitution d'un radical éthyl au radical méthyl du Dimidium. Deux sels d'Ethidium, le chlorure et le bromure ont été synthétisés, qui ont une bonne activité trypanocide. Dans la pratique, seul le bromure d'Ethidium est couramment utilisé.

Le Bromure d'Ethidium ou Bromure d'Homidium est généralement administré aux animaux à raison de $1 \mathrm{mg} / \mathrm{kg}$ de la solution à 1 ou 2 p. 100 par voie intramusculaire profonde. A cette dose, l'Ethidium est actif contre $T$. vivax. L'activité est moins bonne contre $T$. congolense, car on note des rechutes dans certains cas, même avec une dose de $2 \mathrm{mg} / \mathrm{kg}$.

Les traitements effectués par ce produit montrent cependant une plus grande activité et une moindre toxicité que les sels de Dimidium à dose égale. L'Ethidium ne provoque pas la chute de poids, ni la nécrose hépatique périportale que l'on observe avec le Dimidium. De plus, à la dose thérapeutique et par voie intramusculaire, il n'y a pas d'accident de photosensibilisation. Cependant, l'injection du médicament par voie sous-cutanée peut être suivie d'une réaction locale (œdème et nécrose au point d'injection); le lieu d'injection doit être bien massé dans ce cas pour faciliter la dispersion du produit et atténuer la réaction locale.

Chez certains animaux, l'injection d'Ethidium peut être suivie d'une hyperthermie au bout de 48 à 96 heures. Le Cheval semble plus sensible que les Bovins. La toxicité du produit n'est vraiment manifeste qu'à des doses élevées (20).

La principale indication du Bromure d'Ethidium est le traitement curatif de la trypanosomose des Bovins due à $T$. vivax. Le produit peut conférer quelque protection aux animaux traités. Certaines souches de trypanosomes résistent à l'Ethidium, mais cette résistance peut être vaincue par le Bérénil.

\section{c) Le Prothidium ou Pyrithidium}

C'est un dérivé de la Phénanthridine mais structuré selon le plan de l'Antrycide. Il se présente sous forme d'une poudre cristalline de couleur rouge, en partie soluble dans l'eau bouillante. La solution n'est pas stable et le médicament peut précipiter au bout de quelques heures; il convient alors de l'homogénéiser à nouveau par chauffage.

Le Prothidium a une toxicité générale moins grande que les autres dérivés Phénanthridinium. Beaucoup d'auteurs l'ont employé à des doses comprises entre $1 \mathrm{mg}$ et $6 \mathrm{mg} / \mathrm{kg}$ sans observer de réaction générale. Toutefois, l'injection sous-cutanée est toujours suivie de nécrose locale sévère. La voie intramusculaire profonde est la mieux tolérée mais il y a quand même inflammation in situ. 
Administré à raison de $2 \mathrm{mg} / \mathrm{kg}$, le Prothidium est actif contre $T$. congolense, et $T$. vivax chez les Bovins. Utilisé dans des cas de trypanosomose expérimentale des Equidés à $T$. vivax, il a donné de bons résultats (FINELLE, 1965; TOURE, 1968), de même que dans la trypanosomose expérimentale du chien à T. brucei (TOURE, 1970).

Les trypanosomes résistants à l'Antrycide ont tendance à résister en outre au Prothidium. La résistance au Prothidium est souvent accompagnée de résistance croisée avec les autres dérivés Phénanthridinium. Toutes ces résistances sont généralement vaincues par le Bérénil.

\section{d) L'Isométamidium}

Il existe plusieurs sels de Métamidium dont un seul est utilisé dans la pratique : le chlorhydrate de chlorure d'Isométamidium, communément désigné Isométamidium. Ce dérivé est très actif contre les trypanosomes du bétail à des doses de $0,25 \mathrm{mg}$ à $0,8 \mathrm{mg} / \mathrm{kg}$. Sa prescription est courante dans les trypanosomoses des Bovins à $T$. vivax et $T$. congolense. Le produit agit sur $T$. brucei avec cependant des résultats moins constants selon les auteurs. Essayé sur le Chien à raison de $1 \mathrm{mg} / \mathrm{kg}$ pour combattre $T$. brucei, il a permis de juguler assez rapidement la parasitémie tout en étant bien toléré (18).

A des doses comprises entre 0,25 et $0,8 \mathrm{mg} / \mathrm{kg}$, la plupart des animaux supportent la médication par voie intramusculaire. A partir de $1 \mathrm{mg} / \mathrm{kg}$ peuvent apparaître chez les Herbivores des symptômes généraux fugaces. Des doses plus élevées sont certainement toxiques.

Par voie intramusculaire, l'Isométamidium provoque une réaction locale, généralement invisible extérieurement mais qui persiste longtemps et rend impropre à la consommation la viande située autour du point d'injection. Pour pallier cet inconvénient, on peut toutefois administrer l'Isométamidium par voie intraveineuse à raison de $0,25 \mathrm{mg}$ à $0,50 \mathrm{mg} / \mathrm{kg}$ de la solution à 1 p. $100(19)$. A $0,50 \mathrm{mg} / \mathrm{kg}$ par voie intraveineuse, l'efficacité est très bonne et il n'y a pas de réaction générale, ni locale. Mais à $1 \mathrm{mg} / \mathrm{kg}$, la voie intraveineuse est dangereuse et peut être suivie, en quelques heures, de la mort de l'animal traité. La marge de sécurité est assez faible et l'on ne saurait recommander cette voie que si les animaux sont pesés avant le traitement.

L'activité prophylactique du médicament est élevée. C'est au Kenya que les meilleurs résultats ont été obtenus : à la dose de $0,5 \mathrm{mg} / \mathrm{kg}$, voie intramusculaire, la protection conférée peut atteindre 14 semaines, soit plus de trois mois. A la dose de $0,8 \mathrm{mg} / \mathrm{kg}$, il semble raisonnable de compter sur une protection de 3 à 4 mois. Le pouvoir protecteur est conservé par voie intraveineuse et nous avons pu observer chez des Bovins traités à $0,5 \mathrm{mg} / \mathrm{kg}$ une protection de 34 à 49 jours pour T. brucei et 48 à 62 jours pour $T$. vivax.

Les trypanosomes peuvent devenir résistants à l'Isométamidium (21), et il y a possibilité de résistance croisée avec les autres dérivés Phénanthridinium (8). Ces résistances sont vaincues par un traitement au Bérénil.

Dans ces séries de composés actifs contre les trypanosomoses des animaux, les divers médicaments sont appréciés différemment selon les régions, en fonction de la prédominance des espèces de trypanosomes et des types d'élevage. Mais beaucoup des différences d'appréciation, quant à leur efficacité relative, tient certainement dans l'apparition plus ou moins rapide de chimio-résistance.

\section{DE LA CHIMIO-RESISTANCE AUX TRYPANOCIDES}

Bon nombre des trypanocides cités ci-dessus ont un pouvoir curatif immédiat et un pouvoir préventif de plusieurs semaines. Il faut cependant déplorer qu'ils n'offrent pas toujours la garantie absolue de venir à bout de toute infection trypanosomienne, même en les utilisant avec rigueur et attention. En effet, après un emploi plus ou moins prolongé, ces trypanocides 
perdent de leur efficacité vis-à-vis de certaines souches de trypanosomes qui sont devenues chimio-résistantes.

L'apparition de la chimio-résistance tient à une cause essentielle: la concentration du médicament dont on attend l'effet est (ou est devenue) très faible chez l'animal traité. Cela peut tenir aux faits suivants :

1. la concentration efficace n'a pas été atteinte au moment du traitement parce que la dose administrée est plus faible que la dose académique ou bien parce qu'on aura sous-évalué le poids vif de l'animal;

2. il s'est formé un abcès au point d'injection et une partie du médicament a été rejetée avec l'exsudat inflammatoire, ou encore une réaction d'enkystement aura emprisonné le médicament in situ et empêché sa diffusion;

3. un intervalle de temps trop long a séparé deux traitements consécutifs : c'est alors par épuisement du médicament qu'apparaissent des souches chimio-résistantes. Le même phénomène est en cause quand les traitements sont irréguliers ou lorsque les interventions n'ont plus lieu alors que les animaux restent soumis à un risque permanent d'infection;

4. un médicament d'activité préventive a été administré à un animal trypanosomé à la place d'un médicament curatif (surtout dans le cas de la Quinapyramine).

En plus de ces facteurs qui tiennent à la concentration du trypanocide, il en intervient d'autres, liés à l'animal lui-même : la chimio-résistance semble se manifester plus souvent et plus rapidement chez les sujets les plus aptes à fabriquer des anticorps; ils se défendent bien contre les souches résistantes mais celles-ci restent pathogènes pour d'autres animaux.

La résistance croisée est la propriété acquise d'une même souche de trypanosomes de résister à deux ou plusieurs trypanocides. Elle est surtout fréquente avec les trypanocides de même famille chimique : par exemple dérivés Phénanthridinium. Les trypanosomes résistants au Prothidium peuvent résister à l'Antrycide qui est construit sur le même plan que lui. On peut noter aussi une résistance croisée entre deux trypanocides appartenant à deux familles chimiques plus ou moins voisines : c'est le cas notamment pour le Bérénil et l'Antrycide d'un côté, de l'autre l'Antrycide et les dérivés Phénanthridinium.

Le chimio-résistance peut soulever de graves problèmes de thérapeutique quand elle s'est manifestée dans un élevage. Il importe donc de la déceler précocement pour établir un plan d'action rationnel. En tout cas, il est contre-indiqué, lorsqu'une souche résiste à un trypanocide donné, de «forcer la dose » pour la vaincre : cela n'est pas sans danger du point de vue toxicologique, l'effet sur les trypanosomes est aléatoire et le prix des traitements s'en trouve augmenté. Il faut changer de médicament et utiliser celui qui est connu pour être efficace dans le cas considéré.

La résistance à l'Antrycide peut être vaincue sinon par l'Isométamidium, du moins par le Bérénil.

La résistance au Dimidium, à l'Ethidium, au Prothidium ou à l'Isométamidium peut être vaincue par le Bérénil.

La résistance au Bérénil est vaincue par l'Ethidium, le Prothidium, l'Isométamidium et à un degré moindre, par l'Antrycide.

\section{PRATIQUE DES TRAITEMENTS TRYPANOCIDES}

Il est très utile pour le thérapeute sur le terrain de connaître les espèces de trypanosomes à combattre dans les cheptels des différentes régions. La prévalence de l'une des deux espèces les plus courantes ( $T$. congolense ou $T$. vivax) sur l'autre n'a souvent pas d'importance car la plupart des trypanocides modernes sont également actifs à l'égard des deux. Mais selon les 
médicaments dont on dispose, il faudra penser aux différences de sensibilité des espèces de trypanosomes et prévoir, le cas échéant, pour les semaines à venir un produit de substitution. Les infections mixtes par deux espèces de trypanosomes sur un même animal ne sont pas rares dans les régions à glossines. La trypanosomose à $T$. brucei est souvent plus difficile à soigner : il importera de suivre la parasitémie des animaux pour juger des effets de la médication.

Dans les troupeaux à enzootie trypanosomienne, il vaudra mieux dans un premier temps traiter tous les animaux par une dose d'un médicament curatif : Acéturate de Dinamizène ou Bromure d'Ethidium. Une fois l'infection jugulée dans le troupeau, appliquer des mesures prophylactiques dans les deux semaines qui suivent en utilisant un des trypano-préventifs efficaces: Chlorure d'Isométamidium ou Prothidium. Dans ce cas, veiller à la périodicité des traitements prophylactiques, faute de quoi l'on crée des souches résistantes. Si, dans une région fortement infestée de glossines, un éleveur n'est pas à même d'assumer régulièrement les frais de prophylaxie, on peut recommander de n'utiliser que le Bérénil pour traiter les animaux malades afin d'éviter la création de souches résistantes.

Lors de la transhumance saisonnière, certains troupeaux quittent les savanes appauvries pour se diriger vers les pâturages des forêts à glossines; il est alors très indiqué de traiter préventivement les animaux.

Beaucoup de circonspection est requise quand les interventions portent sur des bêtes en mauvais état physiologique et c'est souvent le cas pour les bovins en transhumance, surmenés par la marche. Les injections de trypanocides ne seront pratiquées que sur des bovins reposés et bien abreuvés.

Ne pas traiter par le Dimidium ou l'Ethidium ceux dont le foie fonctionne mal : dans les régions où la fasciolose est fréquente, il faudra une attention particulière. Avec ces deux produits, les animaux à robe claire sont exposés aux accidents de photosensibilisation.

Lorsqu'un animal est destiné à être livré au boucher à échéance plus ou moins brève, il est préférable de le traiter par le Bérénil, d'élimination assez rapide.

Il est toujours indiqué, après un traitement trypanocide, d'améliorer l'état des animaux par un traitement symptomatique. On est tenté, en abordant ce paragraphe, de citer encore, et il y en a beaucoup, des médicaments propres à favoriser le rétablissement des animaux. Mais c'est le régime alimentaire qui doit retenir primordialement l'attention. En certaines régions, fortement infestées de glossines, où plusieurs espèces de trypanosomes pathogènes sont effectivement présentes, mais qui ont des pâturages abondants toute l'année, on constate que la trypanosomose clinique est rare chez les bœufs. En zone de savane, la fréquence des cas cliniques de la maladie est plus grande en saison sèche qu'à la période d'abondance qui suit les pluies, alors même que les populations de Diptères vulnérants sont maximales.

Pour une moindre nocivité des trypanosomoses, il faut donc une bonne alimentation. De même pour rétablir un animal guéri d'une infection, c'est à l'alimentation qu'il faut accorder une attention particulière. Il serait à craindre que cette indication ne fût vaine théorie, tant sont difficiles à résoudre les problèmes d'alimentation du bétail en beaucoup de nos régions. C'est en tout cas, l'occasion de souligner qu'en matière d'élevage, dans les conditions actuelles, c'est la nutrition du bétail qui doit nous préoccuper le plus.

En dehors du régime alimentaire, on peut prescrire certains médicaments pour rétablir les malades après le traitement de l'infection. Il est évident que dans les troupeaux d'élevage traditionnel, la médication symptomatique peut alourdir les frais de l'éleveur. Ce traitement ne sera donc fait que s'il est économiquement justifié par la rentabilité de l'exploitation ou la valeur des animaux (taureaux géniteurs, bœufs de labour, chevaux) ou encore s'il s'agit de favoris domestiques (chiens, chats, moutons d'agrément). Le traitement symptomatique vise à renforcer le cour déficient, à combattre l'anémie, à rétablir l'équilibre des sels minéraux du sérum, accessoirement à améliorer l'appétit. 


\section{SUMMARY}

\section{Trypanocidal drugs as osed in veterinary medicine}

Many species of trypanosomes are involved in animal trypanosomiases whose treatment and chemoprophylaxis are conducted by means of various drugs which are cited for the major. Repeated use of most of these drugs can lead to chemoresistant strains of trypanosomes and this is to be kept in mind when acting with these drugs. In animal treatment it is also important to pay attention to the various species of trypanosomes and to the choice of drugs according to physiological state of animals. This report includes but a few references dealing with general considerations on the subject.

\section{RESUMEN}

\section{Los tripanocidos y su utilización en medicina reterinaria}

Numerosas especies de tripanosomos causan tripanosomiasis en los animales domésticos. Se utiliza, para el tratamiento y la protección de dichos animales, medicamentos varios de los cuales se citan los más corrientes. A la larga, el empleo de estos medicamentos puede provocar la quimioresistencia de cepas de tripanosomos y el terapeuta tiene que poner cuidado en esto. Al fin, convendra tomar en cuenta las varias especies de tripanosomos para el tratamiento de los animales y escoger medicamentos con arreglo al estado fisiologico de los animales.

Este informe no incluye más que un número muy limitado de referencias bibliográficas concemiendo a generalidades sobre el asunto.

\section{BIBLIOGRAPHIE}

1. CURASSON (G.). Traité de protozoologie vétérinaire et comparée. Tome I, Trypanosomiases. Paris, Vigot Frères, 1943, XXXII-445 p. (61 p. de réf.).

2. DAVEY (D.G.). The chemotherapy of animal trypanosomiasis with particular reference to trypanosomal diseases of domestic animals in Africa. Vet. Rev. Annotations, 1957, 3 (1): 15-16.

3. FINELLE (P.). Rapport sur les progrès récents de la chimiothérapie des trypanosomiases animales. Comité scientifique international de Recherches sur les Trypanosomiases, ge réunion, Conakry, 1962, pp. 55-67.

4. FINELLE (P.). Rapport sur la chimio-résistance. Ibid. pp. 101-105.

5. FINELLE (P.) et LACOTTE (R.). Essais de médicaments trypanopréventifs chez les ânes. Comité scient. intern. Rech. Trypanosomiases, 10e réunion, Kampala, 1964, pp. 31-33.

6. GOODWIN (L. G.). The chemotherapy of trypanosomiasis. In : HUTNER (S. H.), Biochemistry and Physiology of Protozoa. New York, Academic Press, 1964. 116 p.

7. ITARD (J.). Toxicologie des injections médicamenteuses utilisées pour le traitement et la prophylaxie des trypanosomiases africaines. Economie Méd. anim., 1965, 6 (4) : 202-212.

8. JONES-DAVIES (W. J.) et FOLKERS (C.). Some observations on cross-resistance to Samorin and Berenil of Homidium resistant strains of Trypanosoma congolense in northern Nigeria cattle. Comité scient. intern. Rech. Trypanosomiases, 1966 , p. 35.

9. MacLENNAN (K. J.R.) and JONES-DAVIES (W.J.). The occurence of a Berenil-resistant Trypanosoma congolense strain in Northern Nigeria, Vet. Rec., 1967, 80 (12): 389-390.

10. MORNET (P.). La lutte contre les trypanosomiases animales en Afrique tropicale. Cah. Méd. vét., 1961, 30 (2): $37-60$.

11. MULLIGAN (H.W.). The African trypanosomiases. London, George Allen et Unwin Ltd, 1970, L XXXVII950 p. (81 p. de réf.).

12. MWAMBU (P.M.) et MAYENDE (P.J.). Detection of strains of T. vivax resistant to Berenil. East african Trypanosomiasis Research Org. Report, 1969, pp. 124-126.

13. NA'ISA (B, K). Follow-up on a survey of the prevalence of Homidium-resistant strains of trypanosomes in cattle in Northern Nigeria and drug cross-resistance tests on the strains with Samorin and Berenil. Bull. epizoot. Dis. Afr., 1967, 15 (3) : 231-234.

14. OGADA (T.). Toxicity of intramuscular Berenil in dogs. East African Trypanosomiasis Research Org. Report, $1969: 21-23$.

15. TOURE (S. M.). Rapports de Protozoologie. In: Rapport sur le fonctionnement pour l'année 1965; 1966; Dakar, Laboratoire national de l'Elevage et de Recherches vétérinaires.

16. TOURE (S. M.). Chimiothérapie et chimioprophylaxie des trypanosomases animales. Dakar, Laboratoire national de l'Elevage et de Recherches vétérinaires, 1967,30 p.

17. TOURE (S. M.). Le Prothidium dans le traitement et la prophylaxie de la trypanosomiase des Equidés. Comité scient. intern. Rech. Trypanosomiases, 12e réunion, Bangui, 1968.

18. TOURE (S. M.). Le Prothidium et l'Isométamidium dans le traitement de la trypanosomiase du chien à Trypanosoma brucei. Rev. Elev. Méd. vét. Pays trop., 1970, 23 (3) : 321-326. 
19. TOURE (S. M.). Note sur l'activité trypanocide de l'Isométamidium administré par voie intraveineuse. Bull. epizoot. Dis. Afr., 1973, 21 (1) : 1-3.

20. UNSWORTH (K.). The curative effect of Ethidium Bromide against Trypanosoma vivax infections of Zebu cattle in West Africa, with observations on the toxicity of the drug. Ann. trop. Med. Parasit., 1954, 48 : 229-236.

21. WHITESIDE (E. F.). Recent work in Kenya on the control of drug resistant cattle trypanosomiasis. Comité scient. intern. Rech. trypanosomiases, 1960, p. 141. 